\title{
Der Einfluss von Neutralsalzen auf das Streckungswachstum der Avena-Koleoptile
}

\section{Doctoral Thesis}

Author(s):

Wuhrmann, Karl

Publication date:

1937

Permanent link:

https://doi.org/10.3929/ethz-a-000099264

Rights / license:

In Copyright - Non-Commercial Use Permitted 


\title{
Der Einfluß von Neutralsalzen auf das Streckungs- wachstum der Avena-Koleoptile
}

\author{
Von der \\ Eidgenössischen Technischen Hochschule in Zürich \\ zur Erlangung der
}

Würde eines Doktors der Naturwissenschaften

genehmigte

Promotionsarbeit

vorgelegt von

Karl Wuhrmann, dipl. rer. nat.

Referent: Herr Prof. Dr. P. Jaccard

Korreferent: Herr Prof. Dr. E. Gäumann

Leipzig

Verlag von Gebrüder Borntraeger

1937 
selben. Diese Quellung, verbunden mit Dispersitätsverminderung, ist durch zahlreiche Versuche sichergestellt. Übereinstimmende Beobachtungen stammen von Strugger (1930) und Wada (1930) an Zellkernen, sowie von Höfler (1928, 1934) bei Kappenplasmolyse von Epidermiszellen der Zwiebelschuppen von Allium cepa. Die Giftwirkung der Salze beruht also auf der Koagulation einzelner Plasmaphasen, wie dies schon Walter (1924) angenommen hat.

Nach den bisherigen Überlegungen müssen die zweiwertigen Ionen dank ihrer großen Ladung die Zelle noch weit heftiger schädigen als die einwertigen, sofern sie überhaupt in den Protoplasten eindringen können. In der Tat er. zeugt Magnesium nach Fig. 6 eine starke Wachstumshemmung der Koleoptilen. Dieses Kation verbindet eben eine relativ große Permeierfähigkeit mit einer starken entladenden Wirkung. Cäsium (Fig. 4) mit einfacher Ladung wirkt des. wegen schädigend, weil es als wenig hydratisiertes Ion eine große Adsorbierbarkeit an die Plasmateilchen besitzt. Der Einfluß der übrigen Erdalkalien beschränkt sich während der verhältnismäßig kurzen Versuchszeit mehr auf die äußersten Plasmagrenzschichten. Aber auch dort können sich starke Koagulationswirkungen geltend machen, wie dies Versuche von Cholodny und Sankewitsch (1934) ergeben. Diese Autoren konnten zeigen, daß nach einer Vorbehandlung von Allium-Zellen mit verdünnten Erdalkalilösungen die Protoplasten in Rohrzucker immer Konkavplasmolyse zeigten. Ca-Lösungen können diese Wirkung noch in 1/1200 n Verdünnung erzeugen.

Diese Versuche beweisen, daß zumindest die äußersten Plasmaschichten eine starke Viskositätserhöhung erfahren. (Führt man die Experimente mit Alkalilösungen durch, so ergibt sich immer Konvexplasmolyse.) Man kann die untersuchten Ionen in bezug auf ihre ,,verhärtende Wirkung " auf das Plasma in folgende Reihe ordnen:

$$
\mathrm{NH}_{4}<\mathrm{Na}<\mathrm{K} \|<\mathrm{Mg}<\mathrm{Ca} \rightarrow \text { Entquellung. }
$$

\section{Zusammenfassung}

1. Die Aufgabe der Arbeit bestand in der Untersuchung des Einflusses einiger Neutralsalze auf das Streckungswachstum der Zellen. Als Versuchsmaterial dienten Avena-Koleoptile.

2. Die Untersuchung setzte ein mit der kolorimetrischen Bestimmung des $\mathrm{K}$-, Mg- und Ca-Gehaltes der Keimlinge, bei Verwendung von verschieden konzentrierten Kulturlösungen der Chloride dieser Kationen.

In einer $25 \mathrm{~mm}$ langen Koleoptilspitze sind nach 72 stündigem Wachstum ungefähr gleichviel Kalium und Magnesiumionen verhanden. Das Kalzium erreicht in dieser Zeit höchstens den dritten Teil der Konzentration der vorstehenden beiden Ionen.

3. Die Streckung von Koleoptiltrommeln in sämtlichen Alkali- und Erdalkalichloriden (ausgenommen $\mathrm{BeCl}_{2}$ ) wird untersucht, wobei heteroauxinhaltige und reine Salzlösungen verwendet werden (Konzentration der Lösungen: 0,01 n).

Das Wachstum von Koleoptilspitzen wird in denselben (reinen) Lösungen untersucht. 
In allen Salzlösungen wird die Streckung der Koleoptilen gegenüber reinem Wasser gehemmt.

4. Die Alkaliionen lassen sich entsprechend ihrer Wirkung nach derselben Reihe ordnen, wie dies in den Versuchen von Pirschle (1932) der Fall war.

5. Die Erdalkaliionen setzen das Wachstum gemäß ihrer Stellung in der lyotropen Reihe herab (starke Giftwirkung von Magnesium!).

6. Analoge Versuche werden in binären und ternären Gemischen von $\mathrm{KCl}, \mathrm{MgCl}_{2}$ und $\mathrm{CaCl}_{2}$ unternommen.

Im Vergleich mit dest. Wasser findet auch in Salzgemischen eine Wachstumshemmung statt.

7. Heteroauxin erhöht die Permeierfähigkeit der Kationen.

8. Die Plastizität und Elastizität der Koleoptile wird durch Kationenadsorption in den Membranen verkleinert.

9. Die plastische Dehnbarkeit der Membranen ist begren. zender Faktor für die Streckung der Zellen.

10. An Hand der Literatur wird der Einfluß der Neutralsalze auf das Plasma untersucht, um die Wachstumshemmung durch Salze auch im Hinblick auf die Veränderungen des Protoplasten durch Kationen zu betrachten.

Die Wachstumshemmung durch Alkaliionen beruht in erster Linie auf ihrer Giftwirkung auf das Plasma. Diejenige durch Erdalkaliionen ist auf ihren Einfluß auf die Membranen zurückzuführen.

Die vorliegende Untersuchung wurde in den Jahren 1935-1937 am Institut für allgem. Botanik an der Eidgenössischen Technischen Hochschule in Zürich durchgeführt. Dem Vorstande des Laboratoriums, Herrn Prof. Dr. P. Jaccard, bin ich für das Interesse, welches er meiner Arbeit stets entgegenbrachte, zu großem Dank verpflichtet. Ebenso möchte ich Herrn Priv.-Doz. Dr. A. Frey-Wyssling für seine Anteilnahme an der Untersuchung bestens danken.

\section{Literatur}

F. Alten, H. Weiland \& E. Knippenberg, Kolorimetrische Ca-Bestimmung über das Pikrolonat. Biochem. Z. 265, 1933.

- — \& B. Kurmies, Kolorimetrische Mg-Bestimmung. Z. angew. Chem. 46, 1933.

_ _ - Vergleichende Untersuchungen zur kolorimetrischen K-Bestimmung. Z. Pflanzenernährung und Düngung 32a, 1933.

K. Aulich, Beiträge zur Kenntnis der Ca-Wirkung auf das Keimlingswachstum von Weizen. Ber. sehw. bot. Ges. 45, 1936.

F. Boas, Untersuchungen über die Mitwirkung der Lipoide beim Stoffaustausch der pflanzlichen Zelle. Biochem. Z. 117, 1921.

J. Bonner, Studies on the growth hormone of plants. Proc. nat. acad. se. 19, 1933.

-, The action of the plant growth hormone. Journ. gen. physiol. 17, 1933.

-, Zum Mechanismus der Zellstreckung auf Grund der Mizellarlehre. Jahrb. wiss. Bot. $82,1935$.

G. A. Borowikow, Über die Ursachen des Wachstums der Pflanzen, I. Biochem. Z. 4x. 1913. 